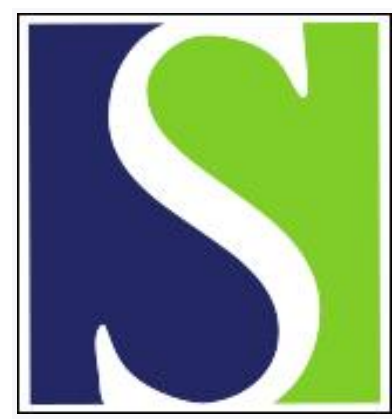

Scand J Work Environ Health 2007;33(1):51-57

https://doi.org/10.5271/sjweh.1064

Issue date: 31 Feb 2007

Simulator driving performance, subjective sleepiness and salivary cortisol in a fast-forward versus a slow-backward rotating shift system

by De Valck E, Quanten S, Berckmans D, Cluydts R

Affiliation: Department of Cognitive and Biological Psychology, Faculty of Psychology and Educational Sciences, Vrije Universiteit Brussel, Pleinlaan 2, B-1050 Brussels, Belgium. elke.de.valck@vub.ac.be

Refers to the following text of the Journal: 2004;30(2):149-156

The following article refers to this text: 2008;34(3):198-205

Key terms: alertness; backward-rotating shift system; circadian rhythm; forward-rotating shift system; salivary cortisol; shift schedule; shift work; simulator driving; sleep; stress; subjective sleepiness; traffic safety

This article in PubMed: www.ncbi.nlm.nih.gov/pubmed/17353965 


\title{
Simulator driving performance, subjective sleepiness and salivary cortisol in a fast-forward versus a slow-backward rotating shift system
}

\author{
by Elke De Valck, PhD, ${ }^{1}$ Stijn Quanten, PhD, ${ }^{2}$ Daniël Berckmans, PhD, ${ }^{2}$ Raymond Cluydts, PhD ${ }^{1}$
}

\begin{abstract}
De Valck E, Quanten S, Berckmans D, Cluydts R. Simulator driving performance, subjective sleepiness and salivary cortisol in a fast-forward versus a slow-backward rotating shift system. Scand $J$ Work Environ Health 2007;33(1):51-57.

Objectives The objectives of this study were to examine simulator driving and subjective sleepiness after morning, afternoon, and night shifts and to compare these differences, as well as objective stress, between a fast-forward and a slow-backward rotating shift system.

Methods The participants were male volunteers working in a chemical plant, 18 in a slow-backward rotating system and 18 in a fast-forward rotating system. All of the participants performed a driving simulator test and subjectively estimated sleepiness after a night, afternoon, and morning shift. Salivary cortisol samples, as indicators of the objective stress level, at the beginning of the workweek —after the second morning shift—were compared between the two rotating shift systems.

Results Lane drifting was higher after a night shift than after an afternoon shift. No effect of rotation system on driving performance could be shown. The subjective sleepiness scores were significantly higher in the slowbackward rotating group than in the fast-forward rotating group. A significant effect of shift type was also observed, with lower levels of sleepiness after the afternoon shift than after the morning and night shifts. Salivary cortisol samples taken at the start of the workweek did not significantly differ between the fast-forward and the slow-backward rotation shift systems.

Conclusions This study indicated that shift type is more important than shift schedule-direction and speed of rotation-in determining driving performance. Performance seemed to be threatened mostly by a night shift and the least by an afternoon shift. In contrast, subjective sleepiness also differed between rotation groups and indicated an advantage of the fast-forward rotation system. The exploratory salivary cortisol measurements suggested that the shift systems studied do not differ in the level of stress they induce, that is to say at the beginning of the workweek.
\end{abstract}

Key terms alertness; circadian rhythm; shift schedule; shift work; sleep; stress; traffic safety.

Rotating shift work has been identified as a risk factor for sleepiness-related traffic accidents in survey (1) and epidemiologic (2) studies. Yet very few experimental and field studies report on the driving performance of shift workers at the end of their shift, when they often drive themselves home by car. Recently, Åkerstedt et al (3) observed a decrease in simulator driving performance after a night shift in comparison with performance after a normal night's sleep.

In our modern industrialized society, a diverse range of shift schedules is applied. In general, a weekly backward (counterclockwise) rotation schedule is considered to be more disadvantageous than a faster forward (clockwise) rotation schedule. Van Amelsvoort et al (4) prospectively related a backward rotating shift system to an increased need for recovery and poor general health, whereas a forward rotation schedule was related to less work-family conflict and better sleep quality over 32 months of follow-up. In line with this result, a questionnaire study among shift workers in the steel industry showed a reduction of social problems when they changed from a weekly backward to a quicker forward rotating shift system (5). The change to the forward rotating schedule was also accompanied by improvement in the satisfaction with the new shift system, whereas no differences were observed concerning general health,

1 Department of Cognitive and Biological Psychology, Faculty of Psychology and Educational Sciences, Vrije Universiteit Brussel, Brussels, Belgium.

2 Department of Biosystems, Division for Measurement, Modelling and Management of Bioresponses, Catholic University of Leuven, Leuven, Belgium.

Reprint requests to: Prof Dr E De Valck, Department of Cognitive and Biological Psychology, Faculty of Psychology and Educational Sciences, Vrije Universiteit Brussel, Pleinlaan 2, B-1050 Brussels, Belgium. [E-mail: elke.de.valck@vub.ac.be] 
sleep quantity and quality, and duration of leisure time. Still, Hakola \& Härmä (6) and Härmä et al (7), comparing two versus three successive night shifts and single versus three successive night shifts, respectively, did observe an advantage for rapidly forward rotating shift systems in comparison with slower backward rotating shift systems in terms of sleep length and sleep efficiency. However, the positive changes in objective sleep were rather small.

Adding to this line of research, the central question in our present study was whether it is more advantageous in terms of driving performance and sleepiness to change shifts rapidly in the course of one workweek (fast-forward rotation shift schedule) or preferable to stay in the same shift during a complete workweek (slow-backward rotation shift schedule). Therefore, we tested shift workers after a morning, an afternoon, and a night shift in the two rotation shift systems, each after an equal number of consecutive workdays. Based on limited previous research on objective driving performance (3) and on psychomotor vigilance performance (8) among shift workers, the hypothesis is that driving performance is generally poorer after a night shift than after an afternoon shift when the longest sleep duration has been observed (8). We are not aware of any published study specifically focusing on the differential influence of a fast-forward versus a slow-backward rotation shift schedule on objectively measured driving performance and could find only one on vigilance performance. Härmä et al (7) showed that changing from a slow-backward to a fast-forward shift system has a significant positive impact on psychomotor vigilance performance, especially after the night shift among older workers, although the effect is minor. In combination with the advantage of the fast-forward system in terms of sleep quality or quantity $(4,6,7)$, this finding leads to the hypothesis that driving performance is less deficient after a night shift in the fast-forward system than in the slow-backward system. Concerning subjective sleepiness, analogous hypotheses are put forward.

In addition, this paper considers an exploratory study of the difference in objective stress in a fast-forward versus a slow-backward rotating shift system. As a marker of objective stress, salivary cortisol levels were measured, as they are considered to reflect hypothalamus-pituitary-adrenal (HPA) axis activity (9). Salivary cortisol has been shown to be a valid measure for monitoring adrenal activity in healthy shift workers as opposed to serum cortisol and can easily be obtained noninvasively (10). Cortisol assessment in a shift system closely resembling the fast-forward rotating system at hand has already indicated a higher total adrenocortical secretion over 24 hours in shift workers than in day workers; this phenomenon suggests higher levels of objective stress in the former. As to a comparison of fast-forward with slow-backward rotating systems, an increased incidence of factors potentially contributing to stress, such as work-family conflict (4) and social problems (5), in the slow-backward systems has been described. This finding raises the hypothesis that employees also generally experience more objective stress in the slow-backward system than in the fast-forward system.

This comparative shiftwork study was carried out in a chemical plant in Belgium, where the fast-forward rotating shift system was introduced a year prior to the study, when the employees could switch to this system voluntarily. Most of the employees who changed to the fast-forward rotating system showed a clear preference for it.

\section{Study population and methods}

\section{Study population}

Thirty-six male volunteers working full-time as technicians in a chemical plant participated in the study. Half of the participants had worked for at least 3 years in a slow-backward rotating system, whereas the other half had started in a fast-forward rotating system 8 to 12 months before the study. In the slow-backward rotating system, employees worked seven consecutive night shifts (2200-0600), which, after 3 days off, were followed by seven consecutive afternoon shifts (1400-2200), which, after 2 days off, were followed by seven consecutive morning shifts (0600-1400). In the fast-forward rotating system, the employees were engaged in the following shifts consecutively: two or three morning shifts (0600-1400), two or three afternoon shifts (1400-2200), and two or three night shifts (2200-0600), in a total of seven successive workdays. The shift systems are graphically presented in figure 1. The mean age of the workers using the slow-backward and the fast-forward rotating systems was 42.3 (SE 1.3)

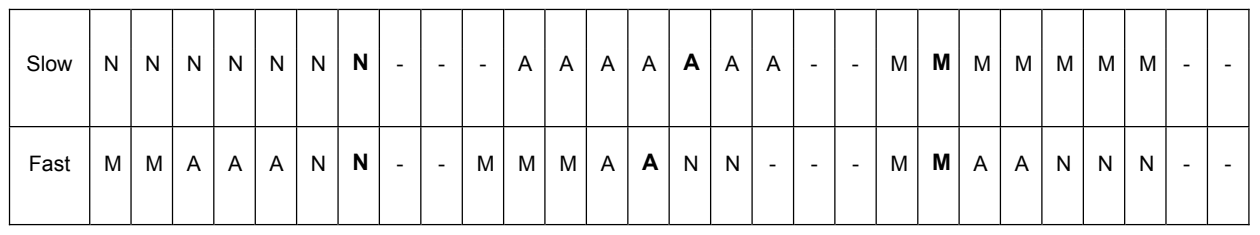

a The shifts in bold were compared between the two rotation systems.
Figure 1. Graphic presentation of the slow-backward and the fast-forward rotating shift systems. ${ }^{a}(\mathrm{~N}=$ night shift, $A=$ afternoon shift, $M=$ morning shift, $-=$ day off) 
years and 42.5 (SE 1.3) years, respectively. The average body mass index (BMI) was 26.21 (SE 0.95$) \mathrm{kg} / \mathrm{m}^{2}$ for the slow-backward group and 27.09 (SE 0.98) kg/m² in the fast-forward group. All of the participants were free of any medication potentially affecting the central nervous system. They gave their informed consent prior to their participation in this study.

\section{Procedure}

The employees were allowed to finish their shift 30 minutes earlier, at 0530, 2130 and 1330, after their night, afternoon, and morning shifts, respectively; the change enabled them to return home at their usual time of day after the completion of the tests. During each testing session, they performed a 25-minute driving simulator test. Before the driving test, they completed subjective sleepiness scales and indicated the number of caffeinated beverages (coffee, tea, cola) they consumed during the preceding shift period. After the driving tests, at 0600, 2200 and 1400 for the night, afternoon, and morning shifts, respectively, salivary cortisol samples were collected and again subjective sleepiness questionnaires were completed. For the analysis of driving performance and subjective sleepiness, the following testing moments were compared: (i) the second morning shift in the slow rotation shift system and the second morning shift in the fast rotation shift system (ie, the second workday in both systems); (ii) the fourth or fifth afternoon shift in the slow rotation shift system and the second afternoon shift in the fast rotation shift system (ie, the fourth or fifth workday in both systems); (iii) the sixth or seventh night shift in the slow rotation shift system and the second night shift in the fast rotation shift system (ie, the sixth or seventh workday in both systems (figure 1). Concerning objective stress, we compared salivary cortisol samples at the beginning of the workweek-after the second morning shift-between the two rotation shift systems.

There was a minimum interval of 1 week between two consecutive testing sessions of an employee.

\section{Design}

All of the participants performed three testing sessions, after the night, afternoon, and morning shifts. The order of participating after a night, afternoon, and morning shift was fully counterbalanced in both the fast-forward and the slow-backward rotating shift groups.

\section{Measurements}

Driving performance. Driving performance was tested using the York Driving Simulator (York Computer Technologies, Kingston, Ontario, Canada) with the Drivesim 3.00 software, running on an IBM-compatible personal computer. The simulated car was equipped with an accelerator, a brake, and a steering wheel. On a 21inch computer screen, a forward view from the driver's seat of a motorway road scene, not including any bends, stop signs or traffic lights, was presented. Speed was displayed at the bottom of the screen. During each driving test, the participants' vehicle was overtaken by two other cars. The driving scenario incorporated artificial wind to make it more difficult for the participants to keep a constant road position and speed. The instruction was to stay in the right-hand lane and to sustain the posted speed of 100 kilometers per hour. The duration of the driving task was 25 minutes. The following driving parameters were calculated: (i) lane drifting, the standard deviation of the road position in an arbitrary lane width of 3 meters calculated in centimeters, (ii) speed deviation, the mean of the differences in kilometers per hour of the speed of the vehicle from the posted speed limit, and (iii) accident liability, being 1 if the car left the road or hit another vehicle at least once during the 25-minute drive, 0 if this was not the case. Speed and road position were sampled at $10 \mathrm{~Hz}$. Data of the first 10 minutes of the drive were excluded from the analyses to allow adaptation to the driving task to develop. As a consequence, all of the analyses are based on the last 15 minutes of the drive.

Subjective sleepiness. Before and after each driving session, subjective sleepiness was assessed with the use of the Stanford Sleepiness Scale (11).

Cortisol. Saliva samples were collected with a Salivette ${ }^{\circledR}$ (Sarstedt, Nümbrecht, Germany), with an insert containing a sterile polyester swab for collection of the saliva, yielding a clear and particle-free sample. The salivettes were used according to the instructions provided by the manufacturer. Samples collected in this way are stable at room temperature for at least a week. The salivettes containing saliva were centrifuged at $2000 \mathrm{~g}$ for 10 minutes, and the filtrates were frozen $\left(-20^{\circ} \mathrm{C}\right)$ and stored. Before the analysis, the samples were thawed and mixed. Saliva cortisol was measured by radioimmunoassay (DiaSorin, Stillwater, MN, USA), using a modification of an unextracted radioimmunoassay method for serum cortisol. Briefly, $200 \mu \mathrm{l}$ of saliva was pipetted into the coated tube and incubated with ${ }^{125} \mathrm{I}$ cortisol for 45 minutes at $37^{\circ} \mathrm{C}$. The modified cortisol assay had a measuring range of $0.5-30 \mu \mathrm{g} / \mathrm{l}$ and within- and between-run coefficients of variation of $<5 \%$ and $<10 \%$, respectively.

As cortisol secretion follows a pronounced circadian rhythm, the sample taken at the end of the second morning shift, at 1400 was compared between the two rotation shift systems. At this moment, all of the employees had worked only two consecutive morning shifts after a minimum of two days off. 


\section{Statistical analysis}

A repeated measures analysis of variance with shift type (night, afternoon, morning) as the within-subject factor and rotation system (slow-backward and fast-forward) as the between-subject factor was performed to analyze driving performance, as measured by lane drifting and speed deviation, salivary cortisol levels, and caffeine consumption, as indicated by the number of caffeinated beverages consumed. For subjective sleepiness (Stanford Sleepiness Scale), an additional within-subject factor, before versus after the driving simulator test, was included. Where appropriate, Tukey posthoc tests were used. For accident liability, a dichotomous variable, Cochran Q-tests and Kolmogorov-Smirnov tests, were performed to assess the effect of shift type and rotating shift system, respectively.

The level of statistical significance was set at the conventional alpha $=0.05$, but we also reported trends (alpha $=0.10)$ because this study is one of the first on the impact of shift work on driving performance.
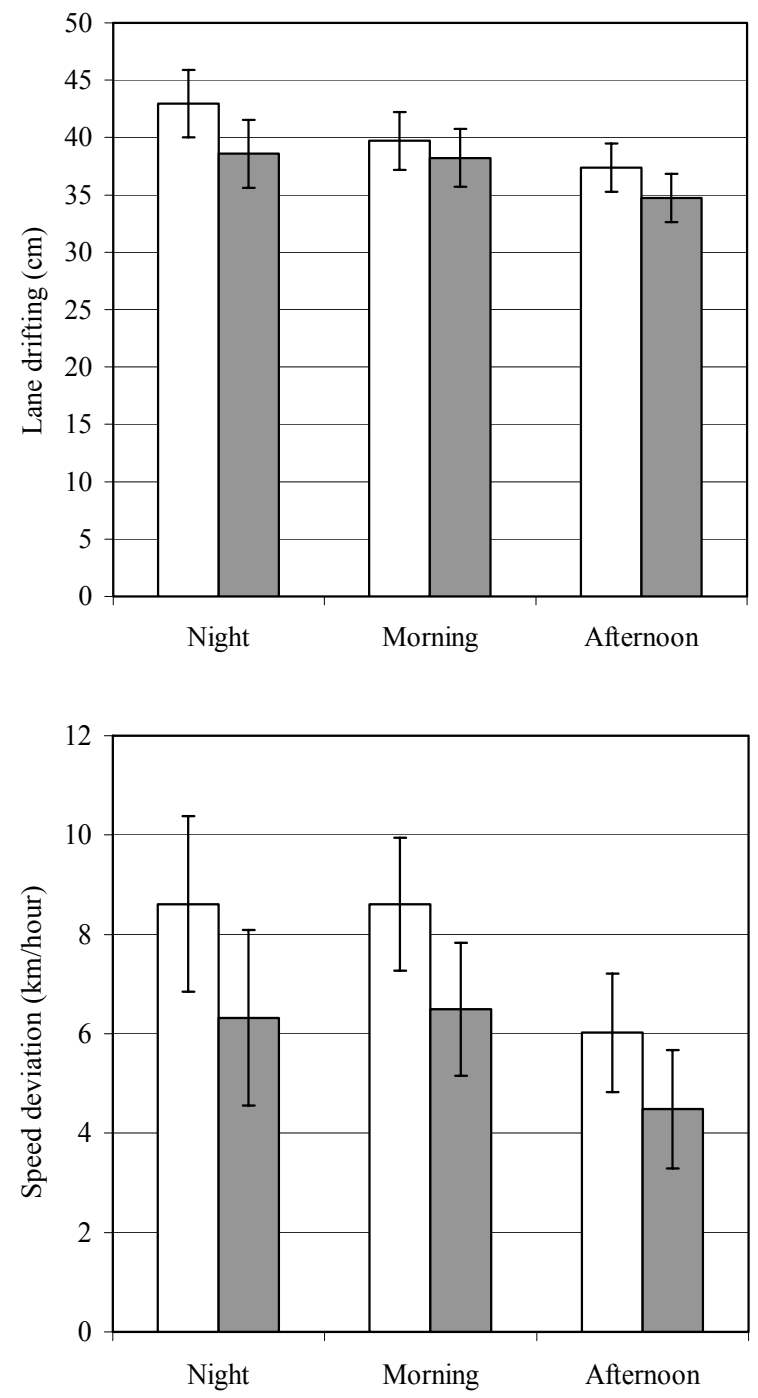

$\square$ Slow backward

$\square$ Fast forward

\section{Results}

\section{Driving performance}

A significant effect of shift type on lane drifting was found $[\mathrm{F}(2,68)=5.79 ; \mathrm{P}<0.01]$. As illustrated in figure 2, posthoc analyses showed that lane drifting was higher after the night shift as compared with the afternoon shift $(\mathrm{P}<0.01)$. No overall effect of rotation system $[F(1,34)=0.81$; not significant $]$ nor a shift type-rotation system interaction $[\mathrm{F}(2,68)=0.53$; not significant $]$ could be demonstrated.

There were no significant main or interaction effects on speed deviation $[\mathrm{F}(2,68)=2.09$; not significant for the effect of shift type, $F(1,34)=1.91$; not significant for the effect of rotation system, $F(2,68)=0.05$; not significant for the interaction effect]. Figure 3 illustrates the findings with respect to speed deviation.

Concerning accident liability, the Cochran Q-tests did not yield a significant effect of shift type, and there was no significant difference between the two rotation $\square$ Slow backward

$\square$ Fast forward

Figure 2. Lane drifting after the night, morning, and afternoon shifts in the slow-backward and the fastforward rotating shift systems. Vertical bars denote standard errors.
Figure 3. Speed deviation after the night, morning, and afternoon shifts in the slow-backward and the fast-forward rotating shift systems. Vertical bars denote standard errors. 
systems, not for the night, the afternoon or the morning shifts as indicated by Kolmogorov-Smirnov tests. A summary of the test results with respect to accident liability can be found in table 1 .

\section{Subjective sleepiness}

Scores on the Stanford Sleepiness Scale were significantly higher in the slow-backward rotating group than in the fast-forward rotating group $[\mathrm{F}(1,31)=6.62 ; \mathrm{P}<0.05]$. A significant main effect of shift type was also observed $[\mathrm{F}(2,62)=19.40 ; \mathrm{P}<0.001]$, the posthoc tests showing a lower level of subjective sleepiness after the afternoon shift than after the morning shift $(\mathrm{P}<0.001)$ and the night shift $(\mathrm{P}<0.001)$. In addition, the scores on the Stanford Sleepiness Scale were significantly increased after the simulator test as compared with before the simulator test $[\mathrm{F}(1,31)=55.58 ; \mathrm{P}<0.001]$. The interaction effect between shift type and before versus after the simulator test $[\mathrm{F}(2,62)=16.82 ; \mathrm{P}<0.001]$ suggested that, before the simulator test, subjective sleepiness was higher after the night shift than after the morning and afternoon shifts, whereas, after the simulator test, the scores were higher after both the night and the morning shifts than after the afternoon shift (all $\mathrm{P}<0.001)$. The difference between the scores of the Stanford Sleepiness Scale before versus after simulator driving was significant after each shift type $(\mathrm{P}<0.001$ for the night and the morning shifts; $\mathrm{P}<0.05$ for the afternoon shift). The other interaction effects were not significant. Figure 4 offers an illustration of these findings.

\section{Salivary cortisol}

The average salivary cortisol level after the second morning shift was 4.91(SE 0.41) $\mu \mathrm{g} / \mathrm{l}$ for the slowbackward rotating group and 4.85 (SE 0.41) $\mu \mathrm{g} / \mathrm{l}$ for the fast-forward rotating group. These cortisol levels did not differ significantly between the shift rotation systems $[\mathrm{F}(1,34)=0.009$; not significant $]$.

\section{Caffeine consumption}

The reported daily consumption of caffeinated beverages did not differ significantly between the fast-rotating $(\mathrm{M}=3.67 ; \mathrm{SE}=0.61)$ and the slow-rotating (mean 3.20; SE 0.62) $[F(1,33)=0.29$; not significant $]$ shift workers. However, there was a significant effect of shift type on caffeine consumption $[\mathrm{F}(2,66)=4.16 ; \mathrm{P}<0.05]$. The participants reported higher caffeine use during the morning (mean 3.76; SE 0.50) and the night (mean 3.57; SE 0.48) shift as compared with the afternoon (mean 2.97; SE $0.42)$ shift $(\mathrm{P}<0.05$ and $\mathrm{P}<0.10$, respectively). There was no significant interaction effect between shift type and rotation system $[F(2,66)=1.61$; not significant $]$.

\section{Discussion}

The main finding of our study was that shift type was more important than shift schedule-direction and speed of rotation-in determining driving performance.

Table 1. Percentage of sessions in which at least one accident occurred among the workers on the slow-backward versus those on the fast-forward rotating shift system after the night, morning, and afternoon shifts.

\begin{tabular}{lccc}
\hline Type of shift & $\begin{array}{c}\text { Slow-backward } \\
\text { system (\%) }\end{array}$ & $\begin{array}{c}\text { Fast-forward } \\
\text { system (\%) }\end{array}$ & $\begin{array}{c}\text { Average } \\
(\%)\end{array}$ \\
\hline Night & 22 & 18 & 20 \\
Morning & 39 & 29 & 34 \\
Afternoon & 22 & 6 & 14 \\
Average & 28 & 18 & 23 \\
\hline
\end{tabular}

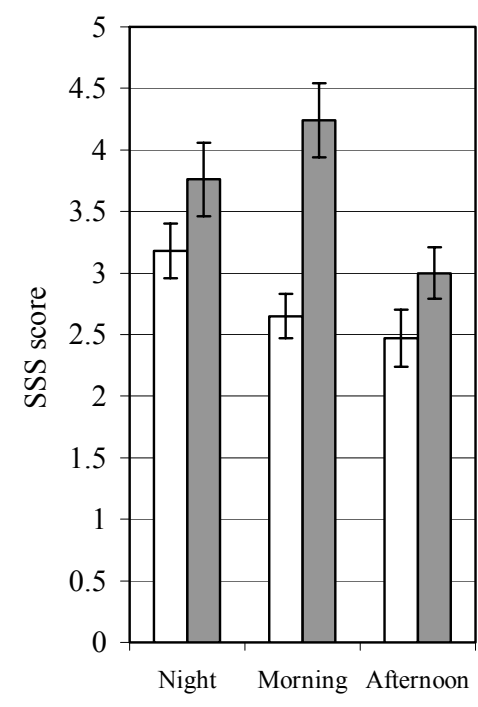

Slow backward

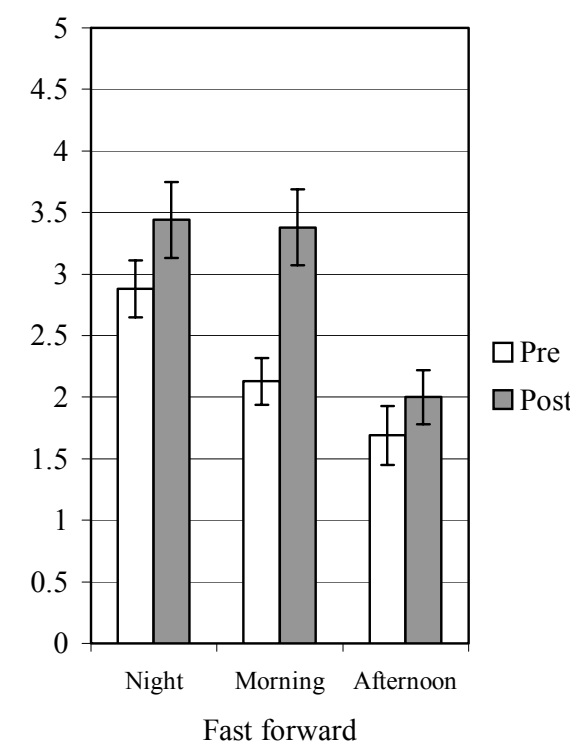

Figure 4. Subjective sleepiness before (pre) versus after the simulator driving test (post) after the night, morning and afternoon shifts in the slow-backward and the fast-forward rotating shift systems. Vertical bars denote standard errors. $($ SSS $=$ Stanford Sleepiness Scale) 


\section{Driving simulator performance}

Driving performance, as indicated by lane drifting, seemed to be threatened mostly by a night shift and the least by an afternoon shift, with the performance after the morning shift falling in between. This finding is in accordance with the finding of increased sleepiness (12) and decreased driving performance (3), as well as decreased psychomotor vigilance performance (8), after a night shift when compared with sleepiness, driving performance and psychomotor performance after the day or afternoon shifts. On one hand, these findings can be related to the reduction of 2-4 hours in sleep duration that is expected after both a night and a morning shift (13). On the other hand, the circadian dip in alertness at night, and to a smaller degree in the afternoon, probably partly explains the performance deficits observed on the simulator.

Although previous studies have supported a preference for a fast-forward as compared with a slow-backward rotation schedule with regard to health, sleep, and social aspects (4-7) and to a limited extent concerning psychomotor performance, driving simulator performance in our study did not significantly differ between the two systems. This finding is in accordance with the results of the laboratory study of Cruz et al $(14,15)$, which indicated that the clockwise direction of rotation does not result in better sleep or vigilance performance than counterclockwise rotation.

It should be mentioned that the participants reported significantly higher caffeine consumption during the morning and night shifts than during the afternoon shift. This result potentially flattened the difference in performance between the shifts, as caffeine positively affects driving simulator performance $(16,17)$.

No significant effects of shift type or shift schedule were found on speed deviation or accident involvement. As reported previously, these driving performance parameters are less sensitive to sleepiness than lane drifting (17).

\section{Subjective sleepiness}

In agreement with Härmä et al (12), subjective sleepiness was clearly higher in the night and the morning shifts than in the day or afternoon shift. Contrary to driving performance, although in line with our expectations, subjective sleepiness also significantly differed between the two rotation groups. The results were suggestive of an advantage for the fast-forward over the slow-backward rotation system concerning the level of subjective sleepiness among the workers. This finding is in accordance with the more deleterious impact of the latter system on sleep quantity and quality, as reported in the literature $(4,6,7)$.

As an advantage of the fast-forward rotation system was only present in terms of subjective measurements, caution is needed when these results are interpreted, especially when the employees' preference for this rotation system is taken into consideration. Moreover, in contrast to our finding, in the large-scale field study of Tucker et al (18), no difference in self-reported sleepiness-alertness was observed between workers in a slow-backward and a fast-forward shift system, which they referred to as an advancing discontinuous and a delaying continuous schedule, respectively.

A comparison of the level of subjective sleepiness before and after the driving test suggests that performing the task either fatigued the employees or made them more aware of their sleepiness, especially after the morning shift.

\section{Salivary cortisol}

To investigate whether one of the shift schedules was more stressful, we compared the cortisol levels after the second morning shift between the two shift systems, ensuring the same time of day for the collection of the samples and an equal opportunity for the adaptation of the employees' endogenous circadian rhythm to the shift schedule. This comparison suggested that there is no preference for a fast-forward over a slowbackward rotating shift system in terms of the level of objective stress, as indicated by the level of salivary cortisol, a marker of HPA axis activity. This finding is in accordance with the finding of Boquet et al (19) indicating that experimentally induced clockwise and counter-clockwise shift schedules result in comparable adrenocortical activity.

However, the work of Boquet et al (19) considered a laboratory study, not addressing long-term effects of forward versus backward rotating shift systems. In addition, it should be stressed that our study considers limited cortisol data. Follow-up studies, using more cortisol samples at regular intervals across the entire circadian cycle are required to enable firmer conclusions on the difference in objective stress between a fast-forward and a slow-backward rotation shift system. In this context, it should be considered that the absence of a difference in objective stress between the two shift systems in our study could be due to the timing of the sampling of the salivary cortisol (ie, after the second morning shift). The research findings of Bonnefond et al (8) suggest that morning shifts are associated with fewer problems in social life, potential sources of stress, than night and afternoon shifts. As such, differences in objective stress between the two systems in our study could have been clearer after a night and an afternoon shift than after the second morning shift.

The strengths of this study are its reasonable sample size, 36 employees, and the counterbalancing of the order of being tested after the night, afternoon, and 
morning shifts, while it was carried out in the workplace. Even though testing shift workers in their natural work environment adds to the external validity of the research findings, we acknowledge that, in field studies, rigid experimental control is not possible, as was clear from the caffeine consumption in the different shifts. In addition, we recognize that changing from the slow-backward to the fast-forward shift rotation system was voluntary. In other words, there was no random assignment of the employees to one of the two shift rotation systems. As Jansen et al (20) showed, this situation must be taken into consideration, as the employees who choose to change to another work schedule can be the ones that experience problems with their work schedule. More generally, the possibility exists that employees in the two different shift systems differed not only in their work schedule, but also in other relevant domains, such as the coping mechanisms they used, like exposure to bright light. For these reasons, intervention studies, instead of cross-sectional studies, are preferable, even though they are not always feasible.

\section{Concluding remarks}

Our results on the driving simulator, as well as with the subjective sleepiness scales, support the guidelines Knauth (21) formulated on the basis of his series of questionnaire studies with shift workers. Indeed, our findings indicate that night work, as well as an early start in the morning, should be avoided as much as possible. In addition, whereas no significant difference in driving performance between the two systems could be illustrated, an advantage of the fast-forward system, as compared with the slow-backward system in terms of subjective sleepiness, was found. This finding corresponds with Knauth's conclusion that forward rotation seems to be the most preferred and that fast-rotating shift systems are preferable to slowly rotating ones.

To end, our study dealt with driving performance, subjective sleepiness, and cortisol in relation to shift work. Evidently, many other issues must be considered in the development of shift schedules, such as social needs of the employees and operational goals of the organization.

\section{References}

1. Gold DR, Rogacz S, Bock N, Tosteson TD, Baum TM, Speizer FE, et al. Rotating shift work, sleep, and accidents related to sleepiness in hospital nurses. Am J Public Health. 1992;82:1011-4.

2. Stutts JC, Wilkins JW, Osberg JS, Vaughn BV. Driver risk factors for sleep-related crashes. Accid Anal Prev. 2003;35:321-31.

3. Åkerstedt T, Peters B, Anund A, Kecklund G. Impaired alert- ness and performance driving home from the night shift: a driving simulator study. J Sleep Res. 2005;14:17-20.

4. van Amelsvoort LGPM, Jansen NWH, Swaen GMH, van den Brandt PA, Kant I. Direction of shift rotation among three-shift workers in relation to psychological health and work-family conflict. Scand J Work Environ Health. 2004;30(2):149-56.

5. Knauth P, Hornberger S. Changes from weekly backward to quicker forward rotating shift systems in the steel industry. Int J Ind Ergon. 1998;21:267-73.

6. Hakola T, Härmä M. Evaluation of a fast-forward rotating shift schedule in the steel industry with a special focus on ageing and sleep. J Hum Ergol. 2001;30:35-40.

7. Härmä M, Hakola T, Kandolin I, Sallinen M, Virkkala J, Bonnefond A, et al. A controlled intervention study on the effects of a very rapidly forward rotating shift system on sleep-wakefulness and well-being among young and elderly shift workers. Int J Psychophysiol. 2006;59:70-9.

8. Bonnefond A, Härmä M, Hakola T, Sallinen M, Kandolin I, Virkkala J. Interaction of age with shift-related sleep-wakefulness, sleepiness, performance, and social life. Exp Aging Res. 2006;32:185-208.

9. Abplanalp JM, Livingston L, Rose RM, Sandwisch D. Cortisol and growth hormone responses to psychological stress during the menstrual cycle. Psychosom Med. 1977;39:158-77.

10. Shinkai S, Watanabe S, Kurokawa Y, Torii J. Salivary cortisol for monitoring circadian rhythm variation in adrenal activity during shiftwork. Int Arch Occup Environ Health. 1993;64:499-502.

11. Hoddes E, Zarcone V, Smythe H, Phillips R, Dement WC. Quantification of sleepiness: a new approach. Psychophysiology. 1973;10:431-6.

12. Härmä M, Sallinen M, Ranta R, Mutanen $P$, Muller K. The effect of an irregular shift system on sleepiness at work in train drivers and railway traffic controllers. J Sleep Res. 2002;11:141-51.

13. Åkerstedt T. Work hours, sleepiness and the underlying mechanism. J Sleep Res. 1995;4 suppl 2:15-20.

14. Cruz C, Boquet A, Detwiler C, Nesthus T. Clockwise and counterclockwise rotating shifts: Effects on vigilance and performance. Aviat Space Environ Med. 2003;74:606-14.

15. Cruz C, Detwiler C, Nesthus T, Boquet A. Clockwise and counterclockwise rotating shifts: Effects on sleep duration, timing, and quality. Aviat Space Environ Med. 2003;74:597605.

16. Reyner LA, Horne JA. Early morning driver sleepiness: effectiveness of $200 \mathrm{mg}$ caffeine. Psychophysiology. 2000;37:251-6.

17. De Valck E, De Groot E, Cluydts R. Effects of slow-release caffeine and a nap on driving simulator performance after partial sleep deprivation. Percept Mot Skills. 2003;96:67-78.

18. Tucker P, Smith L, Macdonald I, Folkard S. Effects of direction of rotation in continuous and discontinuous 8 hour shift systems. Occup Environ Med. 2000;57:678-84.

19. Boquet A, Cruz C, Nesthus T, Detwiler C, Knecht W, Holcomb K. Clockwise and counterclockwise rotating shifts: effects on temperature and neuroendocrine measures. Aviat Space Environ Med. 2004;75:898-904.

20. Jansen NWH, van Amelsvoort LGPM, Kristensen TS, van den Brandt PA, Kant IJ. Work schedules and fatigue: a prospective cohort study. Occup Environ Med. 2003;60 suppl 1:47-53.

21. Knauth P. Designing better shift systems. Appl Ergon. 1996;27:39-44.

Received for publication: 8 May 2006 